Maria do Carmo Leal 1

Célia Landman Szwarcwald 2

\section{Evolução da mortalidade neonatal no Estado do Rio de J aneiro, Brasil (1979-1993): análise por causa segundo grupo de idade e região de residência}

\author{
Evolution of neonatal mortality in the state \\ of Rio de J aneiro, Brazil (1979-1993): \\ an analysis according to causes, by age and \\ region of residence
}

1 Escola Nacional de Saúde Pública, Fundação Oswaldo Cruz.

Av. Leopoldo Bulhões 1480, Rio de Janeiro, RJ, 21041-210, Brasil.

2 Centro de Informações para a Saúde Fundação Oswaldo Cruz. Av. Brasil 4365, Rio de Janeiro, RJ, 21045-900, Brasil.

\begin{abstract}
This paper reports the results of a study on neonatal mortality in the State of Rio de Janeiro (1979-1993), according to major causes of death, by age and regi on of residence. The authors suggest criteria for grouping neonatal deaths according to causes, taking into consideration frequency and preventability. For deaths which occurred in the first day of life, immaturity and fetal growth retardation were the only causes of death that showed significant decline. An increase in deaths due to respi ratory affections of the fetus and newborns was observed. Causes of death said to be reducible by "adequate control of pregnancy and attention at delivery" and "early diagnosis and treatment" remained unaltered. The authors noted that a decrease in death rates took place starting with the 1-6 day age group. The comparison with other countries called attention to the high rates of neonatal death in Rio de Janeiro. It al so pointed out an increase in the proportion of deaths classified as "respi ratory problems of the newborn", demonstrating lack of specificity in defining the basic cause of death.

Key words Neonatal Mortality; Child Health; Mortality; Epidemiology

Resumo Esteartigo apresenta os resultados de um estudo sobre mortalidade neonatal no Estado do Rio de Janeiro (1979-1993), segundo principais grupos de causa de morte, de acordo com idade e regi ão de resi dência. Foi proposto um critério de agrupamento das causas de morte, levando-se em consideração a freqüência das mesmas e a evitabilidade do óbito. Para os óbitos ocorridos no primeiro dia de vida, a imaturidade e crescimento fetal retardado foram as únicas cau sas que mostraram declínio si gni fi cati vo. Houve acentuada el evação do número de mortes por afecções respi ratórias do feto e do recém-nasci do. As causas de óbi to ditas reduzíveis por "adequado controle da gravidez eadequada atenção ao parto" e "diagnóstico e tratamento precoce" permaneceram inalteradas. Constatou-se que o decrésci mo nas taxas ocorreu a partir do grupo etário de 1 a 6 dias. A comparação com outros países chama a atenção para a el evada proporção de óbi tos relaci onadas a "probl emas respiratórios do recém-nasci do", demonstrado falta de especificidade na definição da causa básica deóbito.
\end{abstract}

Palavras-chave Mortalidade Neonatal; Saúde da Criança; Mortalidade; Epidemiologia 


\section{Introdução}

Nas últimas duas décadas, o coeficiente de mortalidade infantil teve evolução de decréscimo nas diversas áreas geográficas do Estado do Rio de Janeiro. Este descenso, no entanto, mostrou-se diferente ao se enfocar o componente precoce ou tardio da mortalidade infantil. Como descrito por Leal \& Szwarcwald (1996), entre 1979 e 1993, foi observada uma queda três vezes maior para a mortalidade pós-neonatal do que para a mortalidade neonatal. Ficou evidente ainda uma tendência de estabilidade do coeficiente nos primeiros sete dias de vida, ocorrendo, inclusive, aumento na mortalidade nas primeiras horas, em torno do momento do nascimento das crianças.

O declive verificado para o comportamento tardio neste período de 15 anos foi expressivo, mesmo quando comparado com a experiência de outros países, evidenciando que a estratégia utilizada foi efetiva e alcançou bons resultados. Por outro lado, a estabilidade da mortalidade infantil precoce e o discreto aumento nas primeiras horas após o parto demonstraram a baixa resolutividade dos programas de pré-natal assim como daqueles de atenção ao parto e ao recém-nascido.

Estes achados relativos à mortalidade neonatal são contrastantes à evolução histórica internacional da mortalidade infantil. De uma maneira geral, países com taxas em nível semeIhante ao do Estado do Rio de Janeiro, alcançaram um enorme decréscimo no componente precoce, mesmo em época anterior, quando não havia se desenvolvido toda a tecnologia perinatal disponível hoje. Já nas décadas de 50 e 60, em países da Europa, no Japão, e mesmo nos Estados Unidos da América, a mortalidade infantil decresceu muito no primeiro mês de vida, em particular na primeira semana, exatamente a correspondente aos dias pós-parto, período em que os recém-nascidos ainda estão sob a responsabilidade dos serviços de saúde (Nakamura et al., 1991; Kalter, 1991; Lee et al., 1980).

Atualmente a mortalidade neonatal se constitui no componente de maior expressão quantitativa da mortalidade infantil no Rio de Janeiro, acarretando desempenho de queda bem aquém do esperado. Pode-se até prever uma estagnação do coeficiente de mortalidade infantil se nenhuma intervenção específica for feita em breve sobre a mortalidade neonatal.

Diferentemente do que se imagina, conforme mostrado por Carvalho (1993), estes óbitos ocorrem por causas evitáveis, que, na sua maioria, dispensam a utilização de tecnologia neo- natal complexa, ou seja, são evitáveis com procedimentos simples, de baixo custo e grande efetividade.

O comportamento inesperado dos coeficientes de mortalidade neonatal no Estado do Rio de Janeiro nos remete à necessidade de conhecer de que causas morrem estas crianças, com vistas à possibilidade de intervir sobre elas. Neste artigo, pretendendo dar uma aproximação a esta questão, analisou-se a distribuição da mortalidade neonatal no Estado do Rio de Janeiro por principais causas de morte, de 1979 a 1993, segundo tempo de vida e região de residência da criança. Buscou-se desenvolver um critério de agrupamento das causas básicas de óbito que levasse em conta, predominantemente, a evitabilidade da morte.

\section{Metodologia}

Para o cálculo do coeficiente de mortalidade infantil no período neonatal, recorreu-se ao Sistema de I nformações sobre Mortalidade do Ministério da Saú de (SIM/MS), como fonte de dados de óbitos, e às Estatísticas do Registro Civil, como fonte de informações sobre os nascimentos. Levando-se em conta que a subnotificação de óbitos no Estado do Rio de Janeiro é de pequena magnitude, o número de óbitos registrados foi considerado igual ao número de mortes ocorridas e utilizado sem qualquer correção.

Já os nascidos vivos tiveram que ser ajustados, devido ao atraso no registro de nascimento. O método de correção baseou-se em estabelecer o número de nascidos vivos para um determinado ano somando-se os nascimentos registrados naquele ano e os registrados em anos posteriores, até oito anos após o nascimento. Todas as etapas do procedimento de ajuste, assim como as estimativas do número de nascidos vivos de 1979 a 1993, encontramse apresentados em Leal \& Szwarcwald (1996).

A série temporal analisada neste trabalho incluiu os anos de 1979 a 1993, período para o qual utilizou-se, no Brasil, a Nona Revisão da Classificação Internacional de Doenças (OMS, 1985). Assim sendo, observa-se que a comparação internacional referiu-se apenas ao período em que se aplicavam as regras da Nona Revisão nos países aqui considerados.

Para análise das tendências temporais, utilizou-se a taxa de variação anual, calculada através de uma regressão exponencial dos coeficientes observados no tempo durante o período de estudo, conforme detal hado em Leal \& Szwarcwald (1996). 
Para o estudo da distribuição dos óbitos neonatais por causa de morte, foi preciso a elaboração de um critério de agrupamento das causas básicas de morte. Buscou-se um critério que levasse em consideração a evitabilidade do óbito ao lado da freqüência de cada causa, tomada segundo a classificação em quatro dígitos.

Como ponto de partida para a elaboração deste critério, considerou-se a classificação idealizada pela Fundação Sistema Estadual de Análise de Dados (Seade) (Seade, 1991), conforme apresentada na Tabela 1.

Constatou-se, entretanto, que este critério de agrupamento não conseguiria elucidar o que vinha ocorrendo com a mortalidade neonatal no Estado do Rio de Janeiro, sobretudo porque grande parte dos óbitos era atribuída a apenas um pequeno número de causas. Isto é, a evolução de cada grupo de causas do Seade era determinada basicamente pelo comportamento de uma ou duas causas predominantes no grupo. Sem entrar na análise dos resultados, o que será deixado para a seção seguinte, apresenta-se a Tabela 3, tão somente com o objetivo de destacar este fato.

Adicionalmente, notou-se que as anomalias congênitas não compõem o elenco dos grupos de causas consideradas como evitáveis pela Fundação Seade. Contudo, como a comparação com outros países mostra uma acentuada redução da mortalidade neste conjunto de causas, julgou-se importante a inclusão deste agrupamento na classificação elaborada neste trabalho.

Foi também necessário modificar os grupos denominados "parcialmente reduzíveis" e "outros reduzíveis". Preferiu-se juntar as deficiências nutricionais (260-269) às diarréias (001009) e pneumonias (480-486), já que, reconhecidamente, estas causa são determinadas pelos mesmos fatores e freqüentemente aparecem em conjunto nos atestados de óbito como causas associadas de morte (Carval ho et al., 1990; Mendonça et al., 1994).

Além disto, não se julgou conveniente considerar os transtornos relacionados à gestação de curta duração e baixo peso ao nascer (765.0 e 765.1) juntos no mesmo agrupamento, uma vez que, segundo as regras de classificação de causa básica do óbito pela Nona Revisão, a causa 765.1 é bem menos específica. O acompanhamento no tempo, como pode ser observado na Tabela 3, mostra que os óbitos classificados como "crescimento fetal retardado e má nutrição fetal" (764) passaram a ser codificados quase sempre como 765.1, a partir da década de 80, justificando-se assim a junção das categorias 764 e 765.1, separadamente da 765.0.
Estas constatações levaram à elaboração de um outro critério de agrupamento das causa básicas de morte, apresentado na Tabela 2. Observa-se que, para não trabal har com um número excessivo de categorias, optou-se por juntar os grupos "reduzíveis por adequado controle da gravidez" e "reduzíveis por adequada atenção ao parto".

\section{Resultados}

Na Tabela 3, estão apresentados os coeficientes de mortalidade infantil entre os menores de sete dias, distribuídos por grupos de causa de morte, segundo o critério de agrupamento da Fundação Seade (Seade, 1991), para anos selecionados entre 1979 e 1993.

A partir da observação dos dados expostos nesta tabela, pode-se destacar a predominância de um número pequeno de causas de morte, que explicam grande parte dos óbitos em cada agrupamento. Nota-se, por exemplo, que um dos grandes grupos "reduzíveis por adequada atenção ao parto" tem cerca de $60 \%$ dos óbitos que o compõem devidos a "hipóxia intra-uterina e asfixia ao nascer", uma das seis causas do conjunto.

Já o grupo das causas "reduzíveis por diagnóstico e tratamento precoce", que apresentava um coeficiente de 38,5/ 100.000 nascidos vivos (NV) em 1979, passando a 60,8 em 1993, teve como principal subcausa "outros problemas respiratórios após o nascimento" (770.8), que congregava, ao início do período analisado, aproximadamente $30 \%$ dos óbitos deste agrupamento e mais de $50 \%$ ao final. Observa-se, assim, que o aclive verificado no grupo deve-se primordialmente ao aumento da classificação nesta rubrica.

Para o acompanhamento da evolução deste pequeno número de causas de morte, que explicam quase que a totalidade dos óbitos no período neonatal precoce, a mortalidade proporcional entre os menores de sete dias, segundo as dez principais causas básicas de morte (desagregadas em quatro dígitos), está apresentada na Tabela 4, para os mesmos anos selecionados anteriormente. O que mais impressiona nestes resultados é a contribuição crescente dos óbitos por "outros problemas respiratórios após o nascimento" (770.8). Esta, abrangendo as crises de cianose sem outra especificação (SOE), desconforto respiratório SOE, insuficiência respiratória SOE e períodos de apnéia SOE originados no período perinatal, foi responsável, em 1979, por cerca de 7\% das mortes, e, em 1993, por quase $1 / 4$ do total. 
Tabela 1

Critérios de evitabilidade de óbitos neonatais da Fundação Seade.

\begin{tabular}{ll}
\hline Grupo de causas & Códigos da CID \\
\hline Reduzíveis por adequado controle da gravidez & $090,760,773,037,771.3$ \\
Reduzíveis por adequada atenção ao parto & $762,763,766,767,768,771$ exceto 771.3 e 771.8 \\
Reduzíveis por diagnóstico e tratamento precoce & $771.8,520-579,770,772,774,777,680-709,775,776,778$ \\
Parcialmente reduzíveis & $260-269,764,765,769$ \\
Outras reduzíveis & $001-009,276,460-519$ \\
\hline
\end{tabular}

Tabela 2

Critério de classificação das causas de morte utilizado neste trabalho.

\begin{tabular}{ll}
\hline Grupo de causas & Códigos da CID \\
\hline 1) Reduzíveis por adequado controle da gravidez e & $090,760,773,037,771.3,762,763,766,767$, \\
por adequada atenção ao parto (exceto 768) & 768,771 (exceto 771.8) \\
2) Hipóxia intra-uterina e asfixia ao nascer & 768 \\
3) Reduzíveis por diagnóstico e tratamento precoce & $771.8,520-579,770$ (exceto 770.8), \\
(exceto 770.8) & $772,774,777,680-709,775,776,778$ \\
4) Outros problemas respiratórios após o nascimento & 770.8 \\
5) Anomalias congênitas & $740-759$ \\
6) Diarréias, Deficiências nutricionais, Pneumonias & $001-009,260-269,480-487$ \\
7) Imaturidade extrema & 765.0 \\
8) Crescimento fetal retardado e má nutrição fetal e & $764,765.1$ \\
outros recém-nascidos de pré-termo & \\
9) Síndrome de angústia respiratória & $769 . X$ \\
10) Sintomas, sinais e afecções mal-definidas & $780-799$ \\
\hline
\end{tabular}

Tabela 3

Coeficientes de mortalidade nos menores de 7 dias (por $10.000 \mathrm{NV}$ ) por causa, segundo o critério de evitabilidade da Fundação Seade. Estado do Rio de J aneiro, 1979-1993.

\begin{tabular}{|c|c|c|c|c|}
\hline Classificação da Fundação Seade & 1979 & 1983 & 1988 & 1993 \\
\hline Reduzíveis por adequado controle da gravidez & 2,7 & 3,1 & 2,7 & 2,6 \\
\hline Reduzíveis por adequada atenção ao parto & 18,3 & 20,6 & 17,1 & 18,1 \\
\hline Hipóxia intra-uterina e asfixia ao nascer (768) & 11,9 & 12,2 & 9,2 & 11,2 \\
\hline Reduzíveis por diagnóstico e tratamento precoce & 38,5 & 60,8 & 67,2 & 60,8 \\
\hline O utros problemas respiratórios após o nascimento (770.8) & 11,3 & 27,6 & 35,2 & 32,7 \\
\hline Parcialmente reduzíveis & 63,5 & 46,2 & 39,9 & 36,1 \\
\hline Crescimento fetal retardado e má nutrição fetal (764) & 3,7 & 0,2 & 0,0 & 0,2 \\
\hline Imaturidade extrema (765.0) & 9,6 & 8,0 & 5,7 & 5,4 \\
\hline O utros recém-nascidos de pré-termo (765.1) & 16,3 & 9,3 & 7,4 & 6,4 \\
\hline Síndrome de angústia respiratória (769.X) & 18,0 & 19,3 & 19,9 & 19,4 \\
\hline O utras reduzíveis & 11,7 & 2,8 & 2,8 & 1,9 \\
\hline Total & 161,0 & 154,4 & 150,2 & 141,2 \\
\hline
\end{tabular}


Mortalidade proporcional (\%) por principais causas (4 dígitos) nos menores de 7 dias. Estado do Rio de J aneiro, 1979-1993.

\begin{tabular}{|c|c|c|c|c|c|c|c|c|}
\hline \multirow[t]{2}{*}{ Causas } & \multicolumn{2}{|c|}{1979} & \multicolumn{2}{|c|}{1983} & \multicolumn{2}{|c|}{1988} & \multicolumn{2}{|c|}{1993} \\
\hline & $(\%)$ & Posto & $(\%)$ & Posto & $(\%)$ & Posto & $(\%)$ & Posto \\
\hline O utros recém-nascidos pré-termo (765.1) & 16,3 & 1 & 9,3 & 3 & 7,4 & 4 & 6,4 & 5 \\
\hline Síndrome de angústia respiratória (769.0) & 11,2 & 2 & 12,5 & 2 & 13,3 & 2 & 13,7 & 2 \\
\hline Imaturidade extrema (765.0) & 9,6 & 3 & 8,0 & 4 & 5,7 & 5 & 5,4 & 6 \\
\hline $\begin{array}{l}\text { O utros problemas respiratórios do recém-nascido } \\
\text { após o nascimento }(770.8)\end{array}$ & 7,1 & 4 & 17,8 & 1 & 23,4 & 1 & 23,2 & 1 \\
\hline Síndrome de aspiração maciça (770.1) & 4,7 & 5 & 4,4 & 7 & 4,6 & 6 & 4,1 & 7 \\
\hline O utras infecções específicas do período perinatal (771.8) & 4,5 & 6 & 6,2 & 5 & 7,7 & 3 & 9,5 & 3 \\
\hline $\begin{array}{l}\text { Sofrimento fetal não especificado } \\
\text { em recém-nascido vivo }(768.4)\end{array}$ & 3,8 & 7 & 3,0 & 8 & 0,1 & 8 & 0,0 & 10 \\
\hline $\begin{array}{l}\text { Asfixia ao nascer não especificada } \\
\text { em recém-nascido vivo (768.9) }\end{array}$ & 3,4 & 8 & 4,8 & 6 & 5,7 & 5 & 6,7 & 4 \\
\hline Anomalias não especificadas do coração (746.9) & 2,7 & 9 & 2,2 & 9 & 2,8 & 7 & 2,6 & 8 \\
\hline Morte sem assistência (798.9) & 2,6 & 10 & 1,4 & 10 & 0,1 & 8 & 0,1 & 9 \\
\hline
\end{tabular}

Ou seja, verifica-se um aumento substancial na proporção de óbitos classificados sem especificidade da causa básica de morte.

De uma maneira geral, as outras causas referentes a problemas respiratórios, como a “síndrome de angústia respiratória" (769.0), a "síndrome de aspi ração maciça" (770.1) e a "asfixia ao nascer não especificada em recém-nascido" (768.9), também mostram situações de aclive, ou, pelo menos, de estabilidade.

Evolução de aumento é ainda encontrada para as "outras infecções específicas do período perinatal" (771.8), que passam da sexta para a terceira posição como causa de morte. Destaca-se que é nesta classificação que são incluídas as "septicemias do recém-nascido", provavelmente, aqui, a causa de maior contribuição.

Pode-se dizer que as únicas causas que realmente mostram decréscimo são as relacionadas à prematuridade (765.1 e 765.0). A primeira causa - "outros recém-nascidos de prétermo" - , que corresponde, em 1979, à prematuridade sem especificação de idade gestacional e/ ou peso, desloca-se para o quinto lugar em 1993, enquanto a “imaturidade extrema” vai da terceira posição para a sexta.

O comportamento temporal das principais causas de óbito entre as crianças de sete a 27 dias está exposto na Tabela 5. Chama a atenção a tendência de declive das "gastroenterites" (001-009), que perdem importância a cada observação no tempo. Iniciando como principal causa, terminam em sétimo lugar. A partir de 1983, o primeiro posto é ocupado pelas “outras infecções específicas do período perinatal", representando $30 \%$ das mortes.

Também nesta faixa etária é perceptível o crescimento da proporção de óbitos classificados como "outros problemas respiratórios após o parto" (770.8), o que surpreende bastante por tratar-se de causa referente ao momento do nascimento da criança.

Como esperado, as "outras afecções e as mal-definidas originadas no período perinatal" (779.9) mostram significativo acréscimo, já que, a partir de 1990, a Secretaria Estadual de Saúde do Rio de Janeiro regulamentou que os estabelecimentos de saúde deveriam declarar como "indefinida" a causa de óbito dos pacientes que falecessem a caminho dos serviços de saúde sem evidência de morte violenta.

A análise da mortalidade neonatal proporcional por causa, segundo região de residência, evidencia o mesmo tipo de comportamento, nas diferentes áreas, com a diminuição da mortalidade por "diarréias, pneumonias e deficiências nutricionais" e por causas referentes à prematuridade e baixo peso ao nascer, ao lado do aumento das causas que demonstram imprecisão na definição da causa básica de morte. Apesar de, na capital, ter sido encontrada uma proporção mais baixa de óbitos classificados como "outros problemas respiratórios após o nascimento" (770.8), há também aumento expressivo nesta categoria, sobretudo no ano de 1988 (Tabela 6).

Agrupando-se as causas de morte segundo o critério apresentado no Tabela 2, analisa-se o comportamento temporal dos coeficientes de 
Mortalidade proporcional (\%) por principais causas (4 dígitos) nos óbitos ocorridos entre 7 e 27 dias de vida. Estado do Rio de J aneiro, 1979-1993

\begin{tabular}{|c|c|c|c|c|c|c|c|c|}
\hline \multirow[t]{2}{*}{ Causas } & \multicolumn{2}{|c|}{1979} & \multicolumn{2}{|c|}{1983} & \multicolumn{2}{|c|}{1988} & \multicolumn{2}{|c|}{1993} \\
\hline & $(\%)$ & Posto & $(\%)$ & Posto & $(\%)$ & Posto & $(\%)$ & Posto \\
\hline Gastroenterite de origem infecciosa presumível (9.1) & 22,8 & 1 & 12,4 & 2 & 7,2 & 3 & 2,4 & 7 \\
\hline O utras infecções específicas do período perinatal (771.8) & 15,2 & 2 & 23,4 & 1 & 28,2 & 1 & 29,7 & 1 \\
\hline $\begin{array}{l}\text { Broncopneumonia devida a microorganismo } \\
\text { não especificado }(485 . X)\end{array}$ & 8,7 & 3 & 8,4 & 3 & 4,2 & 5 & 1,9 & 8 \\
\hline $\begin{array}{l}\text { Pneumonia devido a microorganismo } \\
\text { não especificado }(486 . X)\end{array}$ & 6,1 & 4 & 6,1 & 4 & 9,3 & 2 & 6,9 & 4 \\
\hline O utros recém-nascidos pré-termo (765.1) & 5,4 & 5 & 3,9 & 6 & 4,4 & 4 & 3,0 & 6 \\
\hline Morte sem assistência (798.9) & 4,3 & 6 & 3,8 & 7 & 0,8 & 8 & 0,4 & 9 \\
\hline $\begin{array}{l}\text { Desnutrição protéico-calórica de tipo } \\
\text { não especificado (263.9) }\end{array}$ & 3,5 & 7 & 1,4 & 9 & 0,6 & 9 & 0,4 & 9 \\
\hline $\begin{array}{l}\text { O utros problemas respiratórios do recém-nascido } \\
\text { após o nascimento }(770.8)\end{array}$ & 3,4 & 8 & 4,6 & 5 & 3,0 & 7 & 8,2 & 2 \\
\hline $\begin{array}{l}\text { O utras afecções e as mal-definidas originadas } \\
\text { no perío do perinatal, não especificadas (779.9) }\end{array}$ & 1,4 & 9 & 1,4 & 9 & 3,5 & 6 & 7,2 & 3 \\
\hline Síndrome de angústia respiratória (769.X) & 0,8 & 10 & 2,2 & 8 & 3,0 & 7 & 5,0 & 5 \\
\hline
\end{tabular}

mortalidade neonatal segundo faixa etária (Tabelas 7, 8 e 9). Pelos dados dispostos na Tabela 7, percebe-se que, em 1993, para os óbitos ocorridos no primeiro dia de vida, os grupos mais importantes são “outros problemas respiratórios após o nascimento", "síndrome de angústia respiratória” e “hipóxia intra-uterina”, todos relativos a problemas respiratórios e que conjuntamente correspondem à metade do total. As únicas taxas anuais de decréscimo que mostram-se estatisticamente significativas são as referentes às causas relativas aos recém-nascidos de pré-termo (grupos 7 e 8). O aclive mais significativo deve-se aos "outros problemas respiratórios após o nascimento", com taxa de acréscimo de 5,8\% ao ano. Evidencia-se que as causas ditas "reduzíveis por adequado controle da gravidez e adequada atenção ao parto" não demonstraram progresso algum nos quinze anos analisados.

Para os óbitos de um a seis dias de vida, ao contrário do ocorrido entre os menores de um dia, os resultados da Tabela 8 apontam para decréscimos significativos na rubrica “hipóxia intra-uterina e asfixia ao nascer", cujo coeficiente diminui a uma taxa de $6 \%$ ao ano, e nas causas "reduzíveis por adequado controle da gravidez e adequada atenção ao parto" , com declive anual de 3\%. No entanto, tanto as "reduzíveis por diagnóstico e tratamento precoce", como a "síndrome de angústia respiratória" apresentam comportamento de estabilidade. Já os “outros problemas respi ratórios do recém-nascido" demonstram um aumento expressivo também nesta faixa etária.

Para os óbitos neonatais ocorridos entre as crianças de sete a 27 dias, a tendência de queda é mais acentuada (Tabela 9). Com comportamento semelhante à mortalidade infantil tardia, o subgrupo de maior importância, composto pelas diarréias, deficiências nutricionais e pneumonias, tem declive pronunciado, de $9,2 \%$ ao ano, e é o grande responsável pelo decréscimo da mortalidade nesta faixa etária.

A comparação com outros países está apresentada na Tabela 10 e refere-se ao período de 1980 a 1992. Chama a atenção a diferença entre as magnitudes dos coeficientes de mortalidade neonatal analisados. Em 1979, as taxas do Estado do Rio de Janeiro eram de duas a quatro vezes maiores que as dos Estados Unidos da América, Reino Unido e Japão, e 50\% maior do que a apresentada pelo Chile, em 1981.

Nos períodos de tempo para os quais se coletaram informações, decréscimos importantes são encontrados em todos os países sob consideração, variando de 4\% ao ano, nos Estados Unidos, a 7\% no Japão. O Estado do Rio de Janeiro tem o pior desempenho, com taxa de variação de decréscimo de menos de $1 \%$ ao ano.

É interessante notar que as "anomalias congênitas" constituem-se no único grupo de causas que apresentava, no início dos anos 80, coeficiente da mesma ordem de grandeza dos 
Tabela 6

Mortalidade neonatal proporcional (\%) por grupos de causas e anos selecionados, segundo região de residência.

Estado do Rio de J aneiro, 1979-1993.

\begin{tabular}{|c|c|c|c|c|c|c|c|c|c|c|c|c|}
\hline \multirow[t]{2}{*}{ Grupo de causas } & \multicolumn{4}{|c|}{ Capital } & \multicolumn{4}{|c|}{ Cinturão Metropolitano } & \multicolumn{4}{|c|}{ Interior } \\
\hline & 1979 & 1983 & 1988 & 1993 & 1979 & 1983 & 1988 & 1993 & 1979 & 1983 & 1988 & 1993 \\
\hline Diarréias, Deficiências nutricionais, Pneumonias & 12,6 & 6,0 & 4,6 & 2,9 & 10,3 & 10,0 & 8,8 & 2,8 & 10,3 & 8,3 & 6,9 & 4,1 \\
\hline O utros problemas respiratórios após o nascimento & 5,5 & 11,8 & 17,1 & 12,1 & 6,4 & 16,8 & 20,2 & 25,5 & 5,9 & 15,6 & 18,3 & 23,3 \\
\hline Reduzíveis por diagnóstico e tratamento precoce & 13,6 & 14,8 & 21,9 & 19,4 & 14,3 & 17,1 & 17,5 & 18,3 & 11,6 & 15,2 & 16,0 & 17,5 \\
\hline Crescimento fetal retardado e O utros RN pré-termo & 12,1 & 6,1 & 3,7 & 4,2 & 15,7 & 6,2 & 7,3 & 6,2 & 17,3 & 12,6 & 9,7 & 7,3 \\
\hline Imaturidade extrema & 12,0 & 10,6 & 6,8 & 5,3 & 5,1 & 5,0 & 3,4 & 3,2 & 4,6 & 3,7 & 4,1 & 4,9 \\
\hline
\end{tabular}

Tabela 7

Coeficientes de mortalidade infantil neonatal (por $10000 \mathrm{NV}$ ) nos menores de 1 dia, segundo as principais causas de óbito e respectivas taxas percentuais de variação anual. Estado do Rio de J aneiro, 1979-1993.

\begin{tabular}{lccccccccccccccccc}
\hline Grupo de causas & 1979 & 1980 & 1981 & 1982 & 1983 & 1984 & 1985 & 1986 & 1987 & 1988 & 1989 & 1990 & 1991 & 1992 & 1993 & $t$ \\
\hline $\begin{array}{l}\text { 1) Reduzíveis por adequado } \\
\text { controle da gravidez e parto }\end{array}$ & 5,2 & 5,7 & 6,7 & 6,5 & 6,6 & 5,2 & 5,1 & 5,4 & 5,8 & 6,1 & 6,0 & 7,0 & 6,3 & 6,4 & 6,6 & 0,9 \\
$\begin{array}{l}\text { 2) Hipóxia intra-uterina } \\
\text { e asfixia ao nascer }\end{array}$ & 7,4 & 7,2 & 9,9 & 6,6 & 7,6 & 7,2 & 5,6 & 7,3 & 6,1 & 6,9 & 6,7 & 7,1 & 6,2 & 7,6 & 8,0 & $-0,6$ \\
$\begin{array}{l}\text { 4) Outros problemas respiratórión } \\
\text { após o nascimento }\end{array}$ & 5,3 & 7,9 & 13,8 & 14,8 & 13,5 & 15,6 & 15,4 & 16,9 & 16,7 & 17,8 & 16,4 & 16,6 & 17,0 & 19,0 & 18,0 & $5,8 *$ \\
$\begin{array}{l}\text { 5) Anomalias congênitas } \\
\text { 7) Imaturidade extrema }\end{array}$ & 6,3 & 5,7 & 6,8 & 5,8 & 6,2 & 5,3 & 5,8 & 6,9 & 5,6 & 7,2 & 7,5 & 7,3 & 7,3 & 7,8 & 6,3 & $1,5 *$ \\
$\begin{array}{l}\text { 8) Cresc. fetal retardado } \\
\text { e outros RN pré-termo }\end{array}$ & 10,8 & 15,9 & 11,0 & 8,5 & 8,6 & 7,6 & 6,6 & 7,8 & 7,3 & 6,8 & 7,6 & 6,5 & 6,0 & 6,5 & 6,0 & $-5,0 *$ \\
9) Síndrome de angústia respiratória & 15,1 & 8,1 & 8,4 & 6,6 & 7,7 & 5,9 & 5,6 & 5,6 & 3,7 & 6,1 & 5,4 & 4,4 & 3,7 & 5,8 & 5,2 & $-5,9 *$ \\
& 8,5 & 9,6 & 10,1 & 8,5 & 7,7 & 8,1 & 7,8 & 8,1 & 7,9 & 8,4 & 7,9 & 9,3 & 8,3 & 9,2 & 8,5 & $-0,0$ \\
Total & 75,4 & 76,6 & 83,1 & 71,1 & 70,1 & 65,4 & 63,8 & 70,5 & 65,4 & 72,9 & 70,4 & 74,4 & 69,0 & 75,5 & 71,2 & $-0,4$ \\
\hline
\end{tabular}

t - Taxa \% de variação anual; * - significativo ao nível de 5\%; (1) - segundo definição na Tabela 2.

Tabela 8

Coeficientes de mortalidade neonatal (por 10000 NV) nas crianças de 1 a 6 dias, segundo as principais causas de óbito e respectivas taxas percentuais de variação anual. Estado do Rio de J aneiro, 1979-1993.

\begin{tabular}{lrrrrrrrrrrrrrrrrrrr}
\hline Grupo de causas (1) & 1979 & 1980 & 1981 & 1982 & 1983 & 1984 & 1985 & 1986 & 1987 & 1988 & 1989 & 1990 & 1991 & 1992 & 1993 & $t$ \\
\hline $\begin{array}{l}\text { 1) Reduzíveis por adequado } \\
\text { controle da gravidez e parto }\end{array}$ & 3,9 & 3,9 & 4,8 & 5,2 & 5,0 & 3,6 & 4,9 & 3,9 & 3,5 & 4,4 & 3,5 & 4,1 & 2,9 & 2,9 & 2,9 & $-3,0 *$ \\
$\begin{array}{l}\text { 2) Hipóxia intra-uterina } \\
\text { e asfixia ao nascer }\end{array}$ & 4,5 & 5,0 & 6,5 & 3,9 & 4,6 & 3,9 & 3,2 & 2,9 & 2,0 & 2,3 & 1,4 & 2,6 & 2,6 & 2,8 & 3,2 & $-6,1 *$ \\
$\begin{array}{l}\text { 3) Reduzíveis por diagnóstico } \\
\text { e tratamento precoce }\end{array}$ & 11,8 & 13,0 & 15,2 & 14,4 & 14,4 & 13,2 & 17,5 & 18,2 & 15,8 & 15,0 & 13,3 & 13,7 & 12,9 & 12,6 & 15,4 & 0,2 \\
$\begin{array}{l}\text { 4) Outros probl. respiratórios } \\
\text { após o nascimento }\end{array}$ & 6,0 & 8,9 & 13,6 & 13,7 & 14,1 & 15,0 & 14,6 & 15,3 & 14,1 & 17,4 & 13,2 & 12,3 & 13,9 & 13,2 & 14,7 & $3,0 *$ \\
$\begin{array}{l}\text { 5) Anomalias congênitas } \\
\text { 9) Síndrome de angústia respiratória }\end{array}$ & 7,0 & 9,6 & 10,6 & 12,5 & 10,2 & 11,6 & 11,5 & 11,6 & 11,6 & 16,6 & 11,5 & 8,6 & 10,9 & 9,3 & 10,8 & 10,9 & $-0,4$ \\
Total & 85,6 & 89,9 & 95,7 & 85,7 & 84,3 & 79,3 & 80,4 & 83,4 & 77,9 & 77,3 & 61,8 & 65,4 & 60,7 & 66,2 & 69,9 & $-2,8$
\end{tabular}

t - Taxa \% de variação anual; * - significativo ao nível de 5\%; (1) - segundo definição na Tabela 2. 
Tabela 9

Coeficientes de mortalidade neonatal (por 10000 NV) nas crianças de 7 a 27 dias, segundo as principais causas de óbito e respectivas taxas percentuais de variação anual. Estado do Rio de J aneiro, 1979-1993.

\begin{tabular}{|c|c|c|c|c|c|c|c|c|c|c|c|c|c|c|c|c|}
\hline Grupo de causas (1) & 1979 & 1980 & 1981 & 1982 & 1983 & 1984 & 1985 & 1986 & 1987 & 1988 & 1989 & 1990 & 1991 & 1992 & 1993 & $\mathrm{t}$ \\
\hline $\begin{array}{l}\text { 1) Reduzíveis por adequado } \\
\text { controle da gravidez e parto }\end{array}$ & 2,1 & 1,8 & 1,8 & 2,0 & 1,7 & 1,8 & 1,3 & 2,1 & 1,4 & 1,9 & 1,9 & 1,6 & 0,4 & 0,8 & 0,9 & $-6,7^{*}$ \\
\hline $\begin{array}{l}\text { 3) Reduzíveis por diagnóstico } \\
\text { e tratamento precoce }\end{array}$ & 12,1 & 11,5 & 10,9 & 12,0 & 12,1 & 12,9 & 14,5 & 15,4 & 13,3 & 15,6 & 12,2 & 10,6 & 8,6 & 8,4 & 10,6 & $-1,5$ \\
\hline $\begin{array}{l}\text { 4) O utros probl. respiratórios } \\
\text { após o nascimento }\end{array}$ & 2,3 & 2,5 & 2,8 & 1,7 & 2,1 & 2,0 & 0,5 & 0,6 & 0,7 & 1,9 & 2,4 & 2,4 & 2,5 & 1,7 & 2,7 & 0,1 \\
\hline 5) Anomalias congênitas & 5,1 & 4,5 & 5,2 & 5,3 & 4,9 & 4,2 & 4,3 & 4,1 & 4,3 & 5,5 & 3,8 & 4,6 & 4,2 & 4,4 & 4,4 & $-1,1$ \\
\hline $\begin{array}{l}\text { 6) Diarréias, Deficiências } \\
\text { nutricionais, Pneumonias }\end{array}$ & 28,6 & 23,4 & 19,8 & 15,5 & 13,6 & 12,3 & 10,3 & 11,4 & 10,2 & 11,0 & 7,7 & 5,9 & 4,6 & 4,1 & 4,1 & $-9,2^{*}$ \\
\hline 9) Síndrome de angústia respiratória & a 0,5 & 1,0 & 1,1 & 1,0 & 1,0 & 1,2 & 1,0 & 1,1 & 1,1 & 1,4 & 0,7 & 1,5 & 1,8 & 1,8 & 1,6 & $5,4^{*}$ \\
\hline Total & 67,0 & 59,8 & 55,9 & 50,9 & 45,4 & 46,3 & 44,2 & 44,5 & 39,8 & 48,4 & 35,9 & 34,2 & 29,2 & 29,5 & 32,4 & $-5,3^{*}$ \\
\hline
\end{tabular}

t - Taxa \% de variação anual; * - significativo ao nível de 5\%; (1) - segundo definição na Tabela 2.

outros países, à exceção do Japão. Entretanto, com o avanço da tecnologia, ao lado da existência de rastreamento e aborto de muitas malformações congênitas, em grande parte dos países desenvolvidos, na década de 90 , as taxas mostram declives importantes enquanto a do estado brasileiro permanece praticamente constante.

Comparativamente, apenas os grupos das “diarréias, deficiências nutricionais e pneumonias" e "sinais, sintomas e afecções mal-definidas" mostram diminuição expressiva. Em um comportamento inédito em relação aos demais, o grupo dos óbitos por “hipóxia, asfixia ao nascer e outras afecções respiratórias" apresenta aclive significativo, aumentando anualmente em $2 \%$, enquanto em todos os países anal isados os coeficientes decresceram em mais da metade nos correspondentes períodos de tempo aqui estudados.

\section{Discussão}

Da análise da mortalidade neonatal por causa no Estado do Rio de Janeiro, no período de 1979 a 1993, alguns fatos merecem destaque. O primeiro deles é o baixo desempenho apresentado na redução da mortalidade neonatal precoce em paralelo ao aumento substantivo da proporção de óbitos sem especificação da causa básica, classificados como devidos a "outros problemas respiratários após o nascimento".
Em segundo lugar, chama a atenção que, entre os óbitos ocorridos no primeiro dia de vi$\mathrm{da}$, as causa ditas "reduzíveis por adequado controle da gravidez e adequada atenção ao parto" não demostram tendência de decréscimo, permanecendo no mesmo patamar que no início dos anos 80. I gualmente, entre os óbitos de um a seis dias, o grupo de causas "reduzíveis por diagnóstico e tratamento precoce" apresenta comportamento semelhante.

Uma outra característica observada é o crescimento generalizado da classificação nas causas relacionadas ao aparelho respiratório, que produz verdadeiro impacto ao se comparar o aclive do grupo “hipóxia, asfixia ao nascer e outras afecções respi ratórias" com a evolução francamente decrescente encontrada em outros países.

A falta de especificidade encontrada na classificação das causas de mortalidade neonatal pode ser comparada à ausência de definição das causas de morte em outras faixas etárias. Se, porém, nestas, estas causas compõem grupo único, denominadas de "sintomas, sinais e afecções mal-definidas", no período neonatal, as causas podem estar dispersas em várias rubricas. A inscrição de "insuficiência respiratória aguda”, “insuficiência cárdiovascular", “prematuridade SOE", ou de outros dos termos utilizados no atestado de óbito do recém-nascido, não difere em nada do preenchimento do atestado de óbito de um idoso com a designação de "falência orgânica generalizada”, “senilidade”, etc. 
Coeficientes de mortalidade neonatal (por 10.000 nascidos vivos) por causas (lista B) em países e anos selecionados e no Estado do Rio de J aneiro.

\begin{tabular}{|c|c|c|c|c|c|c|c|c|c|c|}
\hline \multirow[t]{2}{*}{ Causas } & \multicolumn{2}{|c|}{$\begin{array}{l}\text { Estados Unidos } \\
\text { da América }\end{array}$} & \multicolumn{2}{|c|}{ Reino Unido } & \multicolumn{2}{|c|}{ J apão } & \multicolumn{2}{|c|}{ Chile } & \multicolumn{2}{|c|}{$\begin{array}{l}\text { Estado do } \\
\text { Rio de J aneiro }\end{array}$} \\
\hline & 1980 & 1991 & 1980 & 1992 & 1980 & 1992 & 1981 & 1989 & 1979 & 1993 \\
\hline Anomalias Congênitas (740-759) & 149,5 & 101,3 & 173,0 & 78,0 & 64,6 & 61,4 & 169,9 & 188,3 & 133,2 & 125,8 \\
\hline Espinha bífida e Hidroencefalia $(741,742.3)$ & 6,7 & 2,4 & 27,1 & 3,6 & 2,8 & 0,9 & 12,8 & 6,6 & 7,6 & 6,5 \\
\hline $\begin{array}{l}\text { Anomalias Congênitas do coração } \\
\text { e Aparelho Circulatório (745-747) }\end{array}$ & 46,6 & 22,9 & 41,5 & 24,3 & 31,4 & 22,9 & 39,5 & 44,7 & 51,1 & 44,1 \\
\hline $\begin{array}{l}\text { Algumas afecções originadas } \\
\text { no período perinatal (760-779) }\end{array}$ & 530,2 & 336,6 & 410,1 & 360,8 & 307,1 & 101,1 & 795,1 & 476,1 & 1269,0 & 1239,3 \\
\hline Trauma de parto (767) & 19,8 & 2,8 & 20,4 & 11,5 & 23,3 & 9,7 & 40,4 & 13,8 & 7,6 & 12,5 \\
\hline $\begin{array}{l}\text { Hipóxia, Asfixia ao nascer e outras } \\
\text { afecções respiratórias (768-770) }\end{array}$ & 230,6 & 108,5 & 208,2 & 82,5 & 190,4 & 58,6 & 502,3 & 216,5 & 575,2 & 752,1 \\
\hline $\begin{array}{l}\text { Sinais, Sintomas e afecções } \\
\text { mal-definidas (780-799) }\end{array}$ & 4,0 & 4,0 & 2,4 & 2,4 & 0 & 2,6 & 14,7 & 3,3 & 46,8 & 7,2 \\
\hline $\begin{array}{l}\text { Diarréia, Deficiência nutricional } \\
\text { e Pneumonia }(01,19,321)\end{array}$ & 3,4 & 1,1 & 6,0 & 0,8 & 4,3 & 0,7 & 5,2 & 18,2 & 116,6 & 15,8 \\
\hline Todas & 705,8 & 460,6 & 616,0 & 335,7 & 388,0 & 176,2 & 1021,6 & 716,6 & 1610,0 & 1411,6 \\
\hline
\end{tabular}

Constata-se adicionalmente que esta tendência alastra-se por todo o Estado, havendo considerável aumento da proporção de óbitos classificados sem especificação em todas as áreas geográficas que o compõem. Considerando-se a diversidade das condições sócio-econômicas destas regiões, leva-se a pensar que se trata de uma manifestação da dinâmica organizacional dos serviços de saúde, de uma norma não escrita da prestação de serviços que desqualifica a informação sobre os óbitos, dificultando o conhecimento da realidade e a definição de políticas de intervenção adequadas.

É bem verdade que a seleção de uma causa única de morte, que sumarize todo o processo que vai do adoecimento ao óbito, é uma decisão complexa e sujeita à parcialidade e variabilidade na percepção e interpretação do observador (Comstock \& Markush, 1986; Erhardt, 1977; Niobey 1994). Sabendo-se que uma parte substantiva dos óbitos ocorre no primeiro dia de vida, ou mesmo nas primeiras horas após o nascimento, a realidade da atenção ao parto no Estado do Rio de Janeiro, sem uma prática de referenciamento das gestantes e sem o registro da história gestacional, contribui, incontestavelmente, para dificultar o diagnóstico do óbito neonatal.

Seja pelo descaso com a definição da causa de morte ou seja pela dificuldade em dar um diagnóstico do óbito, fato é que pode-se concluir que o traço mais marcante da análise da mortalidade neonatal por causa no Estado do
Rio de Janeiro recai na ausência de especificação da causa básica em parte substancial dos atestados. Esta característica foi aumentando com o tempo e chegou a inviabilizar a utilização de qualquer agrupamento mais criterioso.

Provavelmente, é também por estes motivos que os estudos de validação das estatísticas de mortalidade por causa, no período neonatal, encontram concordâncias tão baixas como as apresentadas por Carvalho \& Si lver (1995) na Região Metropolitana do Rio de Janeiro, e Nobre et al. (1989), em Porto Alegre, no Rio Grande do Sul.

A diminuição expressiva dos óbitos neonatais tardios demonstra a experiência bem sucedida em relação aos programas de controle das diarréias, infecções respiratórias agudas, desnutrição e doenças imunopreveníveis. Por outro Iado, vem a estimular o enfrentamento de um novo desafio, a eleição de novas prioridades programáticas para a redução das mortes evitáveis por "adequado controle da gravidez e adequada atenção ao parto", que não mostraram progresso algum no período analisado.

A persistência da mortalidade neontal precoce em níveis tão elevados não pode ser atribuída à alta complexidade que o enfrentamento desta questão traz aos serviços de saúde, na medida em que os procedimento rotineiros e simples de atenção ao parto não estão sendo praticados (Carvalho, 1993), e nem mesmo o diagnóstico do óbito está sendo aprimorado, como demonstrado neste trabalho. Ao contrá- 
rio, houve uma queda de qualidade que reflete um descompromisso generalizado com a vida, um afastamento preocupante dos serviços de saúde das suas responsabilidades técnicas e sociais.

Ademais, esta piora na qualidade da informação produzida também não foi percebida pelos setores competentes, uma vez que estes indicadores não foram utilizados, como prática de saúde pública, no monitoramento e avaliação dos programas materno-infantis em andamento (Hartz, 1993; Hartz et al., 1996). Por outro lado, a população, não esclarecida quanto aos seus direitos de cidadania, abdicou passivamente do conhecimento da causa de morte das suas crianças, colaborando, com o seu silêncio, para a manutenção deste problema.

\section{Referências}

CARVALHO, M. L., 1993. Mortalidade Neonatal eAspectos da Qualidade da Atenção à Saúde na Região Metropolitana do Rio de Janeiro. Dissertação de Mestrado, Rio de Janeiro: Escola Nacional de Saúde Pública, Fundação Oswaldo Cruz.

CARVALHO, M. L. \& SILVER, L. D., 1995. Confiabilidade da declaração da causa básica de óbitos neonatais: Implicações para o estudo da mortalidade prevenível. Revista de Saúde Pública, 29:342-348.

CARVALHO, M. L.; NIOBEY, F. M. L.; MIRANDA, N. N. \& SABROZA, P. C., 1990. Concordância na determinação da causa básica de óbito em menores de um ano na região metropolitana do Rio de Janeiro. Revista de Saúde Pública, 24:20-27.

COMSTOCK, G. W. \& MARKUSH, R. E., 1986. Further comments on problems in death certification. American Journal of Epidemiology, 124:180-181

ERHARDT, C. L., 1977. The underutilization of Vital Statistics. American Journal of Public Health 67:325-326

HARTZ, Z. M. A., 1993. Evaluation du Programme de Santé Infantile dans une Région du Nort-Est du Brésil. Tese de Doutorado, Montréal: Département d'Administration de la Santé, Faculté de Médicine, Université Montréal.

HARTZZ, M. A.; CHAM PAGNE, F; LEAL, M. C. \& CONTANDRIOPOULOS A. P., 1996. Mortalidade infantil "evitável" em duas cidades no nordeste do Brasil: Indicador de qualidade do sistema local de saúde. Revista de Saúde Pública, 30. (no prelo).

KALTER, H., 1991. Five-decade international trends in the relation of perinatal mortality and congenital malformations: Stillbirth and neonatal death compared. International Journal of Epidemiology, 20:173-179.

LEAL, M. C. \& SZWARCWALD, C. L., 1996. Evolução da mortalidade neonatal no Estado do Rio de Janeiro de 1979 a 1993: Análise por tempo de vida segundo região de residência. Revista de Saúde Pública 30. (no prelo).
LEE, K.; PANETH, N.; GARTNER, L. M.; PEARLMAN, M. A. \& GRUSS, L., 1980. Neonatal mortality: An anlysis of the recent improvement in the United States. American Journal of Public Health, 70:15-21.

LING, E. W.; SOUAN, L. C. \& HALL, J. C., 1991 Congenital anomalies: An increasingly important cause of mortality and workload in a neonatal intensive care unit. American Journal of Perinatology 8:164169.

MENDONÇA, F. E.; GOULART, E. M. A. \& MACHADO, J. A. D., 1994. Confiabilidade da declaração de causa básica de mortes infantis em região metropolitana do sudeste do Brasil. Revista de SaúdePública, 28:385-391.

NAKAMURA, Y.; NAGAI, M. \&YANAGAWA, H., 1991. A characteristic change in infant mortality rate decrease in Japan. Public Health, 105:145-151.

NIOBEY, F. M. L., 1994. A análise da mortalidade infantil pós-neonatal sob a perspectiva das causas múltiplas. Dissertação de Mestrado, Rio de Janeiro: Escola Nacional de Saúde Pública, Fundação Oswaldo Cruz.

NOBRE, L. C.; VICTORA, C. G.; BARROS, F. C.; LOM BARDI, C.; TEIXEIRA, A. M. B. \& FUCHS, S. C., 1989. Avaliação da qualidade da informação sobre a causa básica de óbitos infantis no Rio Grande do Sul (Brasil). Revista de Saúde Pública, 23:207-213

OMS (Organização Mundial da Saúde), 1985. Classificação Internacional de Doenças, Revisão 1975 (Nona Revisão), Vol.1. São Paulo: Faculdade de Saúde Pública, Universidade de São Paulo. Centro da OMS para Classificação de Doenças em Português.

SEADE (Fundação Sistema Estadual de Análise de Dados), 1991. Mortalidade infantil e desenvolvimento. Conjuntura Demográfica, 14/ 15:49-50. 